\title{
СТАБИЛИЗАЦИЯ НЕУСТОЙЧИВЫХ СОСТОЯНИЙ ОБРАТНОГО МАЯТНИКА С ВИНТОВЫМИ ЭЛЕКТРОПРИВОДАМИ
}

\begin{abstract}
Предметом изучения в статье является технологический процесс аналитического синтеза алгоритмов стабилизации неустойчивых состояний обратного маятника с винтовыми электроприводами. Целью является разработка технологии оптимального аналитического синтеза алгоритмов стабилизации неустойчивых состояний обратного маятника с винтовыми электроприводами. Задачи: сформировать физическую модель обратного маятника с винтовыми электроприводами. Разработать с помощью Лагранжевого формализма нелинейную математическую модель обратного маятника с винтовыми электроприводами как объекта автоматической стабилизации. Построить линеаризованную математическую модель объекта автоматической стабилизации в пространстве состояний. Проанализировать функциональные свойства объекта автоматической стабилизации. Сформировать структурные схемы системы автоматической стабилизации состояний обратного маятника с винтовыми электроприводами. Получить аналитические соотношения для вычисления оптимальных значений параметров алгоритмов стабилизации неустойчивых состояний обратного маятника с винтовыми электроприводами. Синтезировать для конкретного обратного маятника с винтовыми электроприводами алгоритм оптимальной стабилизации углового положения. Используемыми методами являются: метод Лагранжа, аналитической линеаризации, пространства состояний, преобразования Лапласа. Получены следующие результаты. Сформирован технологический процесс оптимального аналитического синтеза алгоритмов стабилизации неустойчивых состояний обратного маятника с винтовыми электроприводами. На конкретном числовом примере проиллюстрирована продуктивность предложенного процесса аналитического конструирования и инструментальных средств для параметрического синтеза алгоритмов стабилизации обратного маятника с винтовыми электроприводами. Выводы. Научная новизна полученных результатов состоит в следующем: получены разнотипные модели обратного маятника с винтовыми электроприводами как объекта автоматической стабилизации, отличающиеся от известных учетом динамических свойств винтовых электроприводов, создающих управляющие моменты стабилизации. Сформированы условия выбора параметров пропорционально-дифференциальной структуры алгоритмов стабилизации для обеспечения устойчивости и оптимальности переходного процесса замкнутой системы стабилизации, отличающиеся от известных спецификой формирования управляющих моментов стабилизации по интегральному критерию взвешенного модуля ошибки.
\end{abstract}

Ключевые слова : обратный маятник, винтовой электропривод, объект автоматической стабилизации, алгоритм стабилизации, аналитический синтез, стабилизация неустойчивых состояний, интегральный критерий взвешенного модуля ошибки.

\section{Введение}

Постановка проблемы. Появление во время полета неустойчивых состояний характерно для ряда классов летательных аппаратов. Неустойчивые состояния обусловлены смещением центра давления аэродинамических сил вперед по продольной оси относительно центра масс летательных аппаратов. В различных классах летательных аппаратов известны конструкции, обладающие статической неустойчивостью. Так, для обеспечения маневренности истребителей центр давления аэродинамических сил размещают перед центром масс. На ракеты-носители при полете в атмосфере действует опрокидывающий момент аэродинамических сил. В космическом полете на спутники в условиях отсутствия демпфирующей среды возмущающие воздействия приводят к неустойчивому движению относительно центра масс. Летательный аппарат, находящийся в неустойчивом состоянии, не может быть использован по назначению, так как не выполняется необходимое условие работоспособности - устойчивость $[1,2]$.

Расширение функциональных возможностей летательных аппаратов приводит к усложнению их конструкций и появлению новых неустойчивых состояний в поведении их как объектов автоматического управления. Для стабилизации неустойчивых состояний новых летательных аппаратов необходи- мо изучение причин их появления и моделирование этих явлений с помощью различных инструментальных средств.

Простейшими физическими моделями неустойчивых движений являются различные маятниковые устройства. Использование маятниковых устройств различных конструкций позволяет перенести изучение явлений неустойчивости с проектируемых реальных летательных аппаратов на более простые идеализированные физические модели с целью формирования методов обеспечения стабилизации движений [3 - 6].

В известных моделях маятниковых устройств, как правило, задается только интервал изменения управляющего момента и не учитываются динамические свойства привода, влияющие как на устойчивость, так и на качество автоматической стабилизации.

Использование для создания управляющих моментов винтовых электроприводов, применяемых в ряде перспективных летательных аппаратов, обусловило необходимость проведения исследований по обеспечению стабилизации с их помощью.

Постановка задачи. Разработать технологию синтеза алгоритмов стабилизации, оптимальных по интегральному критерию взвешенного квадрата ошибки, для неустойчивых состояний обратного маятника с винтовыми электроприводами. 


\section{1. Физическая модель обратного маятника с винтовыми электроприводами}

На рис. 1 представлена физическая модель механической части исследуемого маятника в инерциальной системе координат OXY.

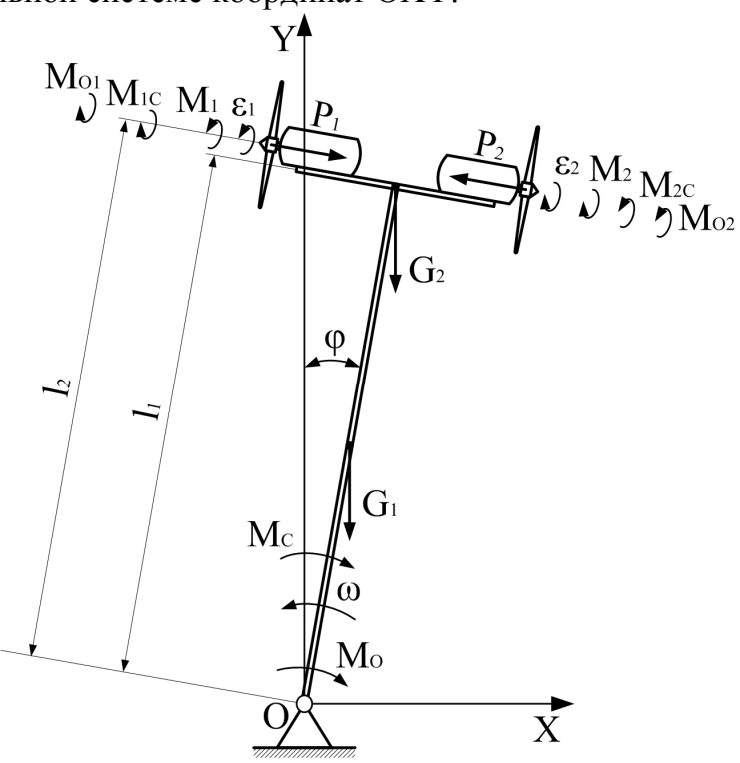

Рис. 1. Физическая модель маятника:

$\varphi, \varepsilon_{1}, \varepsilon_{2}-$ угловые положения маятника, первого и второго винтов; $\omega$ - угловая скорость маятника;

$G_{1}-$ вес стержня; $G_{2}-$ вес поперечной балки;

$P_{1}, P_{2}$ - тяги винтов; $M_{1}, M_{2}$ - вращающие моменты

электроприводов; $M_{c}, M_{c 1}, M_{c 2}$ - моменты аэродинамического сопротивления маятника и винтов; $M_{o}, M_{o 1}, M_{o 2}$ - статические моменты сопротивления

в шарнире мятника и опорах электроприводов

На маятник действуют следующие силы и моменты: вес стержня $G_{1}$; вес поперечной балки $G_{2}$; тяги первого и второго винтов $P_{1}$ и $P_{2}$; вращающие моменты электроприводов $M_{1}$ и $M_{2}$; момент аэродинамического сопротивления маятника $M_{c}$; моменты аэродинамического сопротивления винтов $M_{c 1}$ и $M_{c 2}$; статический момент сопротивления в шарнире маятника $M_{o}$; статические моменты сопротивления в опорах электроприводов $M_{o 1}$ и $M_{o 2}$. Параметры углового движения: угловое положение $\varphi$ и угловая скорость $\omega$ маятника; угловые положения $\varepsilon_{1}, \varepsilon_{2}$ и угловые скорости винтов $\omega_{1}$ и $\omega_{2}$. Физическая модель винтового электропривода показана на рис. 2 .

Особенность данного электропривода заключается в создании основного магнитного поля с помощью постоянных магнитов, установленных на внутренней поверхности статора. В результате взаимодействия постоянного магнитного поля статора с электрическим полем якоря в обмотке якоря возникает движущий момент $M_{i}, i=\overline{1,2}$ и индуктируется противоЭДС $e_{я i}, i=\overline{1,2}$. Часть подведенного на- пряжения $u_{R i}, i=\overline{1,2}$ рассеивается в виде тепла на активном сопротивлении $R_{g i}, i=\overline{1,2}$ обмотки.

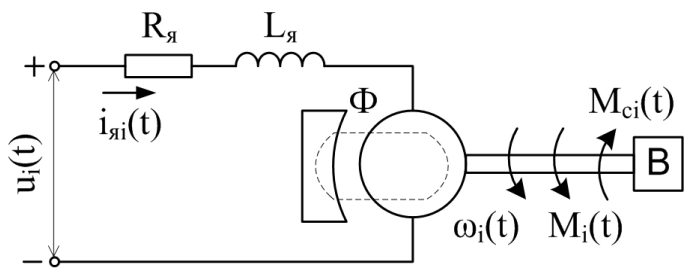

Рис. 2. Физическая модель винтового электропривода: $u_{i}(t), i=\overline{1,2}$ - управляющее напряжение;

$i_{\text {gi }}(t), i=\overline{1,2}$ - ток якоря; $\omega_{i}(t), i=\overline{1,2}-$ угловая

скорость вращения якоря с винтом; $\Phi$ - магнитный поток; $R_{я}$ - сопротивление обмотки якоря;

$L_{я}-$ индуктивность обмотки якоря

Параметрами движения в обмотках якорей первого и второго электроприводов являются заряды $q_{1}, q_{2}$ и токи $i_{1}$ и $i_{2}$; угловые положения $\varepsilon_{1}, \varepsilon_{2}$ и угловые скорости винтов $\omega_{1}$ и $\omega_{2}$.

\section{2. Нелинейная математическая модель}

Для формирования математической модели исследуемого маятника используем уравнения Лагранжа второго рода [7] в следующем виде:

$$
\frac{d}{d t}\left(\frac{\partial T}{\partial q_{i}}\right)-\frac{\partial T}{\partial q_{i}}=Q_{i}^{r}+Q_{i}^{u} ; i=\overline{1, n},
$$

где Т - выражение кинетической энергии объекта моделирования; $\mathrm{q}_{\mathrm{i}}$ - обобщенная координата; $Q_{i}^{r}-$ обобщенная сила, соответствующая силам сопротивления; $Q_{i}^{u}$ - обобщенная сила, соответствующая управляющим воздействиям.

Движение маятника характеризуется пятью степенями свободы, поэтому в качестве обобщенных координат выберем $\varphi, \varepsilon_{1}, \varepsilon_{2}, q_{1}, q_{2}$.

Кинетическая энергия обратного маятника с винтовыми электроприводами определяется таким выражением:

$$
\mathrm{T}=\frac{1}{2}\left(I_{n p} \varphi^{2}+I_{p}\left(\varepsilon_{1}^{2}+\varepsilon_{2}^{2}\right)+L_{\Omega}\left(q_{1}^{2}+q_{2}^{2}\right)\right),
$$

где $I_{n p}=\left(\frac{4}{3} m_{1}+m_{2}\right) l_{1}^{2}-$ приведенный момент инерции маятника; $m_{1}$ - масса стержня; $m_{2}$ - масса поперечной балки с электроприводами; $I_{p}$ - момент инерции ротора электропривода с винтом; $L_{\Omega}-$ индуктивность обмотки якоря.

Обобщенная сила $Q_{i}^{r}$, найденная посредством формирования выражения элементарной работы, описывается следующим образом:

- для обобщенной координаты $\varphi$ :

$$
Q_{\varphi}^{r}=-M_{o}-M_{c} ;
$$


- для обобщенной координаты $\varepsilon_{1}$ :

$$
Q_{\varepsilon_{1}}^{r}=-M_{o 1}-M_{c 1} ;
$$

- для обобщенной координаты $\varepsilon_{2}$ :

$$
Q_{\varepsilon_{2}}^{r}=-M_{o 2}-M_{c 2} ;
$$

- для обобщенной координаты $q_{1}$ :

$$
Q_{q_{1}}^{r}=-e_{\Omega 1}-u_{R 1}
$$

- для обобщенной координаты $q_{2}$ :

$$
Q_{q_{2}}^{r}=-e_{я 2}-u_{R 2} .
$$

Обобщенная сила $Q_{i}^{u}$ будет равна:

- для обобщенной координаты $\varphi$ :

$$
Q_{\varphi}^{u}=P_{1} l_{1}-P_{2} l_{2}+\frac{1}{2} G_{1} l_{1} \sin \varphi+G_{2} l_{1} \sin \varphi ;
$$

- для обобщенной координаты $\varepsilon_{1}$ :

$$
Q_{\varepsilon_{1}}^{u}=M_{1}
$$

- для обобщенной координаты $\varepsilon_{2}$ :

$$
Q_{\varepsilon_{2}}^{u}=M_{2}
$$

- для обобщенной координаты $q_{1}$ :

$$
Q_{q_{1}}^{u}=u_{1} ;
$$

- для обобщенной координаты $q_{2}$ :

$$
Q_{q_{2}}^{u}=u_{2} .
$$

Выполним операции над полученными выражениями в соответствии с уравнением Лагранжа (1):

$$
\left\{\begin{aligned}
& I_{n p} \varphi=P_{1} l_{2}-P_{2} l_{2}+\left(G_{1} l_{1} \sin \varphi\right) / 2+ \\
&+G_{2} l_{1} \sin \varphi-M_{o}-M_{c} ; \\
& I_{p} \varepsilon_{1}=M_{1}-M_{o 1}-M_{c 1} ; \\
& I_{p} \varepsilon_{2}=M_{2}-M_{o 2}-M_{c 2} ; \\
& L_{g 1} q_{1}+u_{R 1}+e_{g 1}=u_{1} ; \\
& L_{g 2} q_{2}+u_{R 2}+e_{g 2}=u_{2} .
\end{aligned}\right.
$$

Аэродинамические моменты сопротивления $M_{c}, M_{c 1}, M_{c 2}$ определяются следующими соотношениями:

$$
\begin{gathered}
M_{c}=m_{c}(\varphi) \frac{\rho l_{1}^{2} \varphi^{2}}{2} S_{b} l_{1} ; \\
M_{c i}=m_{c i}\left(\varepsilon_{i}\right) \frac{\rho r_{b}^{2} \varepsilon_{i}^{2}}{2} S_{b} r_{B} ; i=\overline{1,2},
\end{gathered}
$$

где $m_{c}(\varphi), m_{c i}\left(\varepsilon_{i}\right)$ - аэродинамические коэффициенты; $\rho$ - плотность воздуха; $r_{b}$ - радиус винта; $S_{b}-$ ометаемая площадь винта. Сила тяги винтовых электроприводов характеризуется таким соотношением:

$$
P_{i}=\alpha \rho \omega_{i}^{2} d_{B}^{4} ; i=\overline{1,2},
$$

где $\alpha-$ коэффициент тяги винта; $d_{b}$ - диаметр винта.
Вращающий момент электропривода постоянного тока определяется таким выражением:

$$
M_{i}=n_{i} q_{i} B_{i} a_{i} \sin \left(\varepsilon_{i} t\right) ; i=\overline{1,2},
$$

где $n_{i}$ - число витков обмотки якоря; $B_{i}$ - магнитная индукция; $a_{i}$ - площадь витка; $t$ - время.

Противо-ЭДС, индуктируемая в обмотке якоря при его вращении с угловой скоростью $\varepsilon_{i}$, определяется таким соотношением:

$$
e_{g i}=\varepsilon_{i} r_{g i} B_{i} l_{n i} \sin \left(\varepsilon_{i} t\right) ; i=\overline{1,2},
$$

где $r_{я i}$ - радиус якоря; $l_{n i}$ - длина проводника, охватываемого магнитным полем.

Падение напряжения на активном сопротивлении обмотки якоря характеризуется выражением:

$$
u_{R i}=R_{i} q_{i} .
$$

Уравнения (13) - (19) представляют собой математическое описание нелинейных движений обратного маятника под действием возмущающих и управляющих воздействий. Использование нелинейной математической модели для достижения поставленной цели не представляется возможным, поэтому получим упрощенную математическую модель.

\section{3. Линеаризованная математическая модель}

Для упрощения нелинейной математической модели использовался метод аналитической линеаризации, основанной на разложении уравнений (13) (19) в ряд Тейлора относительно начальных условий, соответствующих вертикальному положению маятника, и пренебрежении членами более высокого порядка малости. В результате получена система:

$$
\left\{\begin{array}{l}
a_{11} \Delta \varphi+a_{12} \Delta \varphi+a_{13} \Delta P_{1}+a_{14} \Delta P_{2}=0 ; \\
a_{21}^{\prime} \Delta \varepsilon_{1}+\Delta \varepsilon_{1}=b_{21}^{\prime} \Delta q_{1} ; \\
a_{31}^{\prime} \Delta \varepsilon_{2}+\Delta \varepsilon_{2}=b_{31}^{\prime} \Delta q_{2} ; \\
a_{41}^{\prime} \Delta q_{1}+\Delta q_{1}=b_{41}^{\prime} \Delta \varepsilon_{1}+b_{42}^{\prime} \Delta u_{1} ; \\
a_{51}^{\prime} \Delta q_{2}+\Delta q_{2}=b_{51}^{\prime} \Delta \varepsilon_{2}+b_{52}^{\prime} \Delta u_{2} ; \\
a_{61}^{\prime} \Delta \varepsilon_{1}=-\Delta P_{1} ; \\
a_{71}^{\prime} \Delta \varepsilon_{2}=-\Delta P_{2},
\end{array}\right.
$$

где $a_{11}=I_{n p} ; a_{12}=-\left(\frac{1}{2} G_{1}+G_{2}\right) l_{1} \cos \varphi_{0} ; a_{13}=-l_{2}$; $a_{21}^{\prime}=I_{p}\left(\frac{\partial M_{1}}{\partial \varepsilon_{1}}+\frac{\partial M_{c 1}}{\partial \varepsilon_{1}}\right)_{0}^{-1} ; b_{21}^{\prime}=\left(\frac{\partial M_{1}}{\partial q_{1}}\right)_{0}\left(\frac{\partial M_{1}}{\partial \varepsilon_{1}}+\frac{\partial M_{c 1}}{\partial \varepsilon_{1}}\right)_{0}^{-1} ;$ $a_{14}=l_{2} ; \quad a_{31}^{\prime}=I_{p}\left(\frac{\partial M_{2}}{\partial \varepsilon_{2}}+\frac{\partial M_{c 2}}{\partial \varepsilon_{2}}\right)_{0}^{-1} ; \quad a_{41}^{\prime}=\frac{L_{я 1}}{R_{я 1}} ;$ $b_{31}^{\prime}=\left(\frac{\partial M_{2}}{\partial q_{2}}\right)_{0}\left(\frac{\partial M_{2}}{\partial \varepsilon_{2}}+\frac{\partial M_{c 2}}{\partial \varepsilon_{2}}\right)_{0}^{-1} ; \quad b_{41}^{\prime}=-\frac{1}{R_{я 1}} \frac{\partial e_{я 1}}{\partial \varepsilon_{1}} ;$ $b_{42}^{\prime}=\frac{1}{R_{я 1}} ; \quad a_{51}^{\prime}=\frac{L_{я 2}}{R_{я 2}} ; b_{51}^{\prime}=-\frac{1}{R_{я 2}} \frac{\partial e_{я 2}}{\partial \varepsilon_{2}} ; \quad b_{52}^{\prime}=\frac{1}{R_{я 2}} ;$ $a_{61}^{\prime}=-2 \alpha \rho \varepsilon_{10} d_{b}^{4} ; a_{71}^{\prime}=-2 \alpha \rho \varepsilon_{20} d_{b}^{4}$. 
Коэффициенты $a_{21}^{\prime}, a_{31}^{\prime}, a_{41}^{\prime}$ и $a_{51}^{\prime}$ отражают инерционные свойства первого и второго электроприводов. Учитывая, что $a_{11}^{\prime} a_{41}^{\prime}$ и $a_{31}^{\prime} a_{51}^{\prime}$, и введя новые обозначения, систему уравнений (20) можно записать в более простом виде:

$$
\left\{\begin{array}{l}
a_{11} \Delta \varphi+a_{12} \Delta \varphi+a_{13} \Delta P_{1}+a_{14} \Delta P_{2}=0 \\
a_{21} \Delta \varepsilon_{1}+\Delta \varepsilon_{1}=b_{21} \Delta u_{1} \\
a_{31} \Delta \varepsilon_{2}+\Delta \varepsilon_{2}=b_{31} \Delta u_{2} \\
a_{41} \Delta \varepsilon_{1}=-\Delta P_{1} \\
a_{51} \Delta \varepsilon_{2}=-\Delta P_{2}
\end{array}\right.
$$

где $\quad a_{21}=T_{1} ; \quad b_{21}=\kappa_{1} ; \quad a_{31}=T_{2} ; \quad b_{31}=\kappa_{2}$; $a_{41}=-2 \kappa_{4} \varepsilon_{10} ; a_{51}=-2 \kappa_{5} \varepsilon_{20} ; T_{1}, T_{2}$ - постоянные времени электроприводов с учетом моментов инерции винтов; $\kappa_{1}, \kappa_{2}-$ коэффициенты передачи электроприводов; $\kappa_{4}, \kappa_{5}$ - коэффициенты преобразования винтов. Представим систему уравнений (21) в следующих переменных состояния:

$$
\Delta \varepsilon_{1}=x_{1}(t) ; \Delta \varepsilon_{2}=x_{2}(t) ; \Delta \varphi=x_{3}(t) ; \Delta \varphi=x_{4}(t) .
$$

В результате соответствующих преобразований получим в векторно-матричной форме уравнение:

$$
\begin{aligned}
& {\left[\begin{array}{l}
x_{1}(t) \\
x_{2}(t) \\
x_{3}(t) \\
x_{4}(t)
\end{array}\right]=\left[\begin{array}{cccc}
-1 / a_{21} & 0 & 0 & 0 \\
0 & -1 / a_{31} & 0 & 0 \\
0 & 0 & 0 & 1 \\
\frac{a_{13} a_{41}}{a_{11}} & \frac{a_{14} a_{51}}{a_{11}} & -\frac{a_{12}}{a_{11}} & 0
\end{array}\right]\left[\begin{array}{l}
x_{1}(t) \\
x_{2}(t) \\
x_{3}(t) \\
x_{4}(t)
\end{array}\right]+} \\
& +\left[\begin{array}{cc}
b_{21} / a_{21} & 0 \\
0 & b_{31} / a_{31} \\
0 & 0 \\
0 & 0
\end{array}\right]\left[\begin{array}{l}
u_{1}(t) \\
u_{2}(t)
\end{array}\right] ;\left[\begin{array}{l}
x_{1}\left(t_{0}\right) \\
x_{2}\left(t_{0}\right) \\
x_{3}\left(t_{0}\right) \\
x_{4}\left(t_{0}\right)
\end{array}\right]=\left[\begin{array}{c}
0 \\
0 \\
x_{30} \\
x_{40}
\end{array}\right] .
\end{aligned}
$$

Для того, чтобы конструкцию обратного маятника с винтовыми электроприводами можно было использовать как объект автоматической стабилизации, её необходимо дополнить датчиком угла $\varphi-$ углового положения. Тогда выходной сигнал датчика $u_{\partial}$ будет связан через коэффициент передачи $\kappa_{\partial}$ с вектором состояния следующим образом:

$$
u_{\partial}(t)=\left[\begin{array}{llll}
0 & 0 & \kappa_{\partial} & 0
\end{array}\right]\left[\begin{array}{l}
x_{1}(t) \\
x_{2}(t) \\
x_{3}(t) \\
x_{4}(t)
\end{array}\right],
$$

а уравнения (22), (23) можно представить в виде

$$
x(t)=A x(t)+B u(t) ; x\left(t_{0}\right)=x_{0} ; u_{\partial}(t)=c^{m} x(t)
$$

как линеаризованную модель объекта автоматической стабилизации.

\section{4. Анализ функциональных свойств объекта автоматической стабилизации}

Необходимыми условиями для формирования алгоритмов управления объекта автоматической стабилизации являются: 1) возможность управлять состояниями объекта с помощью используемых винтовых электроприводов; 2) возможность наблюдать состояния посредством измерения состояния $x_{3}(t)$. Возможность управления состояниями объекта автоматической стабилизации оценим с помощью критерия управляемости Р. Калмана применительно к системе (24) в такой форме:

$$
\operatorname{rang} R=\left[\begin{array}{llll}
B & A B & A^{2} B & A^{3} B
\end{array}\right] \text {. }
$$

Сделав соответствующие преобразования с матрицами $A$ и $B$, получим матрицу управляемости размера $\operatorname{dim} R=4 \times 6$. Среди столбцов этой матрицы можно найти четыре столбца следующей структуры, сведенные в подматрицу $R_{1}$ :

$$
R_{1}=\left[\begin{array}{cccc}
r_{11} & 0 & r_{15} & 0 \\
0 & r_{22} & 0 & r_{26} \\
0 & 0 & r_{35} & r_{36} \\
0 & 0 & r_{45} & r_{46}
\end{array}\right] .
$$

Определитель подматрицы $R_{1}$ :

$$
\operatorname{det} R_{1}=r_{11} r_{22}\left(r_{35} r_{46}-r_{45} r_{36}\right) .
$$

Если раскрыть структуру коэффициентов $r_{i j}$, то получим следующее выражение:

$$
\operatorname{det} R_{1}=\frac{b_{21}^{2} b_{31} b_{41}}{a_{11}^{2} a_{21}^{2} a_{31}}\left(\frac{a_{13} a_{14} a_{51} b_{31}-b_{21} a_{11} a_{21} a_{31}}{a_{21} a_{31}}\right) .
$$

Для полной управляемости объекта автоматической стабилизации по Калману необходимо и достаточно, чтобы $\operatorname{det} R_{1} \neq 0$. В нашем случае это означает, что $a_{13} a_{14} a_{51} b_{31} \neq b_{21} a_{11} a_{21} a_{31}$.

Подставив конкретные выражения параметров, получаем условие полной управляемости:

$$
2 l_{2}^{2} \kappa_{4} \varepsilon_{20} \kappa_{2} \neq \kappa_{1} I_{n p} T_{1} \kappa_{2},
$$

т.е. линейно независимы столбцы, а следовательно, ранг матрицы $R$ rang $R=4$ и объект автоматической стабилизации является полностью управляемым, т.е. он может быть переведен из некоторого начального состояния $x\left(t_{0}\right)$ в желаемое состояние $x\left(t_{1}\right)$ за конечный интервал времени $t_{1}-t_{0}$.

Возможность наблюдения состояний объекта автоматической стабилизации оценим с помощью критерия наблюдаемости Р. Калмана. Для системы уравнений (24) требуется сформировать следующую матрицу наблюдаемости:

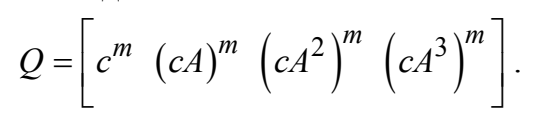

Выполнив соответствующие преобразования матриц $c$ и $A$, получим матрицу наблюдаемости $Q, \operatorname{dim} Q=4 \times 4$. Необходимое и достаточное условие наблюдаемости по Калману заключается в том, чтобы ранг матрицы $\mathrm{Q}$ был равен размерности вектора состояния $x(t)$, т.е. 4. Для выполнения этого 
условия требуется, чтобы $\operatorname{det} Q \neq 0$. Определитель матрицы наблюдаемости равен

$$
\operatorname{det} Q=\kappa_{\partial}^{4} \frac{a_{13} a_{41} a_{14} a_{51}}{a_{11}^{2}}\left(-\frac{1}{a_{21}}+\frac{1}{a_{31}}\right) .
$$

В физических параметрах объекта автоматической стабилизации получим такое выражение:

$$
\operatorname{det} Q=-\kappa_{\partial}^{4} \cdot 4 l_{2}^{2} \kappa_{3} \kappa_{4} \varepsilon_{10} \varepsilon_{20} / I_{n p}^{2} \cdot\left(-1 / T_{1}+1 / T_{2}\right) \text {. }
$$

Полученное выражение не равно нулю, если

$$
1 / T_{1} \neq 1 / T_{2} .
$$

При этом условии объект автоматической стабилизации будет полностью наблюдаем, что означает возможность вычислять все переменные состоя- ния. Практическое выполнение условия (32) означает одновременное использование различных по динамическим свойствам винтовых электроприводов. Если винтовые электроприводы используются не одновременно, а попеременно, то для полной наблюдаемости двумерного вектора состояния объекта автоматической стабилизации можно использовать идентичные электроприводы.

Итак, объект автоматической стабилизации состоит из обратного маятника, двух винтовых электроприводов и датчика углового положения маятника. В линейном приближении процесс преобразования управляющих воздействий $u_{1}(t)$ и $u_{2}(t)$ в выходной сигнал датчика угла $u_{\partial}(t)$ графически можно представить с помощью структурной схемы (рис. 3).

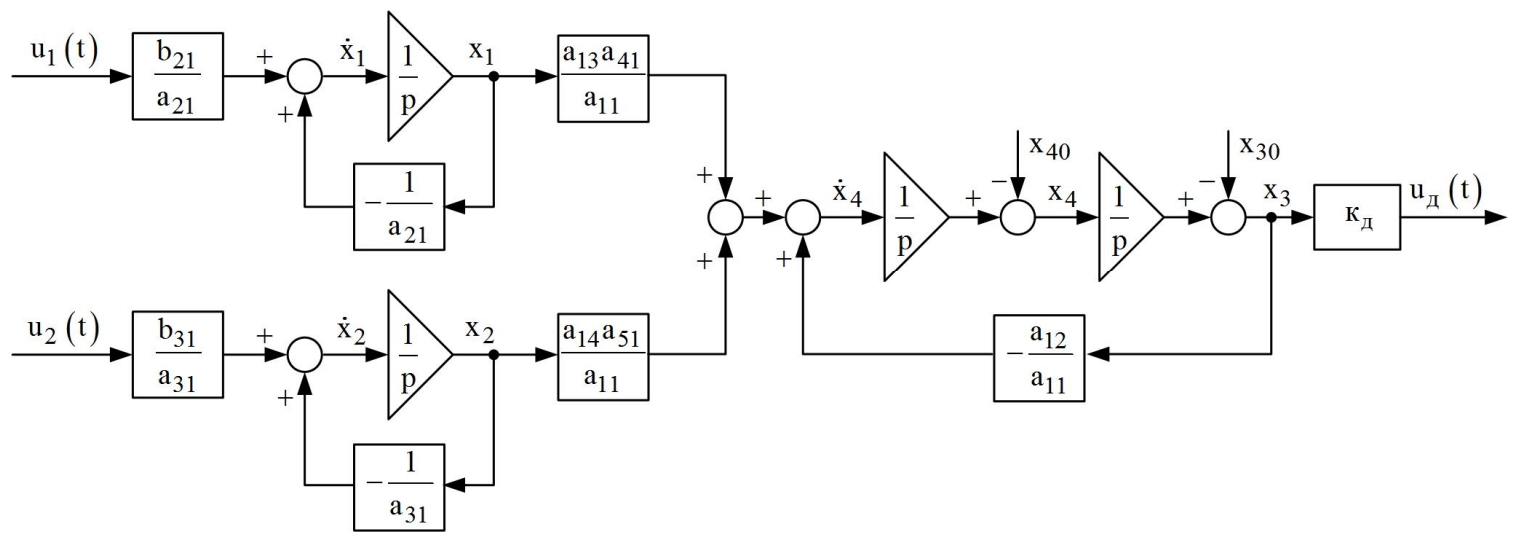

Рис. 3. Структурная схема линеаризованной модели объекта автоматической стабилизации; $\frac{1}{p}-$ символ интегрирования

\section{5. Синтез системы автоматической стабилизации}

Продуктивность решения задачи стабилизации обратного маятника существенно зависит от правильного выбора её структуры. Рассматриваемый объект автоматической стабилизации имеет три степени свободы и четырехмерный вектор состояния. Чтобы обратный маятник сохранял заданное положение равновесия, требуется измерять все переменные состояния и результаты измерений использовать для формирования управляющих воздействий $u_{1}(t)$ и $u_{2}(t)$ на винтовые электроприводы.

В исследуемой конструкции используется только датчик углового положения обратного маятника, измеряющий переменную состояния $x_{3}(t)=\varphi(t)$. Для обеспечения недостающей информации о состоянии обратного маятника, а именно, о переменной состояния $x_{4}(t)$ в структуру алгоритма стабилизации можно ввести дифференцирующую составляющую, формирующую сигнал о скорости изменения углового положения. На рис. 4 представлена структурная схема с использованием передаточных функций.
В схеме используются следующие обозначения: $\kappa_{3}$ - коэффициент передачи сигнала углового положения; $\kappa_{4}-$ коэффициент передачи о скорости изменения углового положения; sign - переключательная функция; $U_{3}(s)$ - изображение задающего воздействия; $E(s)$ - изображение сигнала ошибки; ОАС - объект автоматической стабилизации; УАС устройство автоматической стабилизации.

Передаточная функция замкнутой системы по управляющему воздействию при использовании первого винтового электропривода будет иметь вид:

$$
\begin{gathered}
\Phi(s)=U_{\partial}(s) / U_{3}(s)= \\
=\kappa_{1} \kappa_{\partial} /\left(T_{1} s^{3}+s^{2}+\left(\kappa_{1} \kappa_{\partial} \kappa_{4}-c T_{1}\right) s+\kappa_{1} \kappa_{\partial} \kappa_{3}-c\right)
\end{gathered}
$$

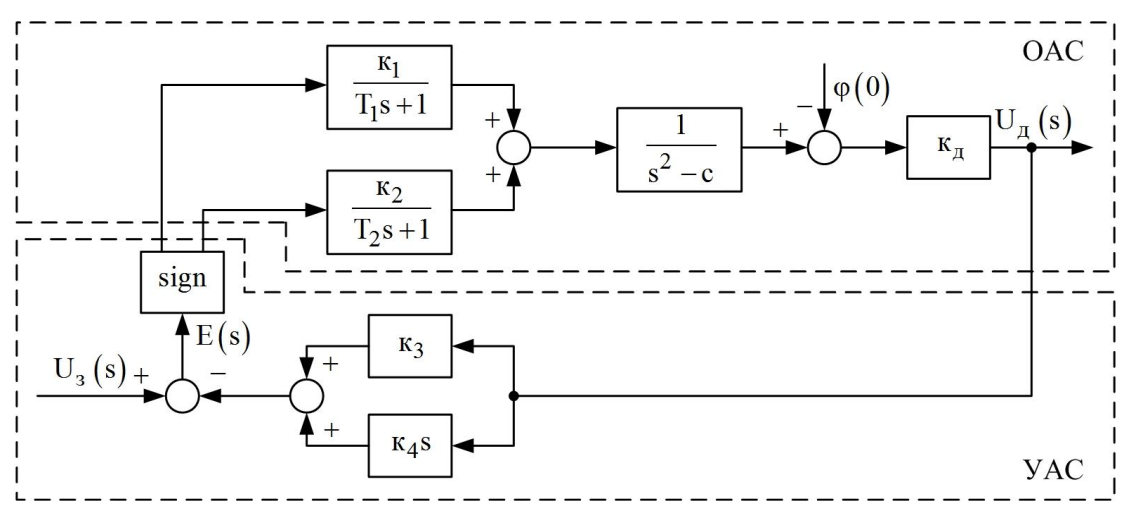

Рис. 4. Структурная схема системы автоматической стабилизации 
Два параметра $\kappa_{3}$ и $\kappa_{4}$ в передаточной функции (33) неизвестны. Выбор этих параметров будем производить исходя из двух условий работоспособности системы: 1) необходимого - обеспечения устойчивости функционирования замкнутого контура системы; 2) достаточного - обеспечения оптимальных показателей качества функционирования системы стабилизации.

Для выполнения необходимого условия работоспособности воспользуемся критерием Гурвица для характеристического уравнения системы

$$
T_{1} s^{3}+s^{2}+\left(\kappa_{1} \kappa_{\partial} \kappa_{4}-c T_{1}\right) s+\kappa_{1} \kappa_{\partial} \kappa_{3}-c=0 .
$$

Необходимое условие устойчивости по критерию Гурвица - положительность коэффициентов характеристического уравнения, следовательно, нужно так выбирать параметры, чтобы

$$
\kappa_{4}>\frac{c T_{1}}{\kappa_{1} \kappa_{\partial}} ; \kappa_{3}>\frac{c}{\kappa_{1} \kappa_{\partial}} .
$$

Достаточное условие устойчивости

$$
\kappa_{1} \kappa_{\partial} \kappa_{4}-c T_{1}-T_{1}\left(\kappa_{1} \kappa_{\partial} \kappa_{3}-c\right)>0
$$

позволяет определить колебательную границу устойчивости

$$
\kappa_{4}=T_{1} \kappa_{3} .
$$

Область устойчивости в системе координат приведена на рис. 5.

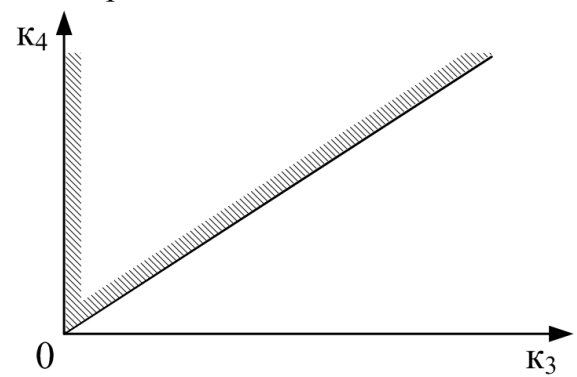

Рис. 5. Область устойчивости системы стабилизации

Из выражения (37) следует, что область устойчивости зависит от инерционных свойств винтового электропривода. Чем больше инерционность, т.е. $T_{1}$, тем меньше область устойчивости. С уменьшением $T_{1}$ область устойчивости расширяется.

Итак, выбор параметров УАС из области устойчивости обеспечивает необходимое условие работоспособности системы стабилизации положения обратного маятника. Конкретизировать выбор можно введением новых условий, сужающих область устойчивости. Такие условия формируются из достаточных условий работоспособности, т.е. требований к качеству переходного процесса.

Для обеспечения достаточного условия работоспособности воспользуемся интегральным критерием качества

$$
I=\int_{0}^{T} t|e(t)| d t
$$

который определяется как интеграл от взвешенного модуля ошибки на интервале времени $[0 ; T]$. Выбор параметров $\kappa_{3}$ и $\kappa_{4}$ из условия обеспечения минимума критерия (38) обеспечит оптимальность показателей качества стабилизации положения обратного маятника относительно выбранного критерия. По критерию (38) определены для передаточной функции замкнутой системы оптимальные значения коэффициентов, которые минимизируют ошибку $e(t)$ в реакции на ступенчатое управляющее воздействие $[8,9]$. Для системы третьего порядка передаточная функция замкнутой системы должна иметь следующий вид:

$$
\Phi(s)=\frac{U_{\partial}(s)}{U_{3}(s)}=\frac{\omega_{n}^{3}}{s^{3}+1,75 \omega_{n} s^{2}+2,15 \omega_{n}^{2} s+\omega_{n}^{3}},
$$

где $\omega_{n}-$ средний геометрический корень стандартного полинома.

Для того, чтобы привести исходную передаточную функцию (33) к требуемому виду (39) введем предшествующий фильтр с передаточной функцией

$$
W_{\phi}(s)=\frac{U_{3}^{\prime}(s)}{U_{3}(s)}=\kappa_{3}-\frac{a_{12}}{\kappa_{1} \kappa_{\partial}} .
$$

Структурная схема системы автоматической стабилизации преобразуется к виду на рис. 6 .

Передаточная функция замкнутой системы в соответствии со структурной схемой (рис. 6) и передаточной функцией (39) будет иметь такой вид:

$$
\begin{gathered}
\Phi_{1}(s)=U_{\partial}(s) / U_{3}(s)= \\
=\frac{\left(\kappa_{1} \kappa_{\partial} \kappa_{3}-c\right) / T_{1}}{s^{3}+s^{2} / T_{1}+\left(\kappa_{1} \kappa_{\partial} \kappa_{4}-c T_{1}\right) \cdot s / T 1+\left(\kappa_{1} \kappa_{\partial} \kappa_{3}-c\right) / T_{1}} .
\end{gathered}
$$

Сопоставляя коэффициенты передаточных функций (41) и (39), получим такие соотношения:

$$
\omega_{n}=\frac{1}{1,75 T_{1}} ; \kappa_{4}=\frac{T_{1}\left(2,15 \omega_{n}^{2}+c\right)}{\kappa_{1} \kappa_{\partial}} ; \kappa_{3}=\frac{T_{1} \omega_{n}^{3}+c}{\kappa_{1} \kappa_{\partial}},
$$

с помощью которых вычисляются значения параметров $\kappa_{3}$ и $\kappa_{4}$, обеспечивающие оптимальность переходного процесса в соответствии с интегральным критерием взвешенного модуля ошибки (38).

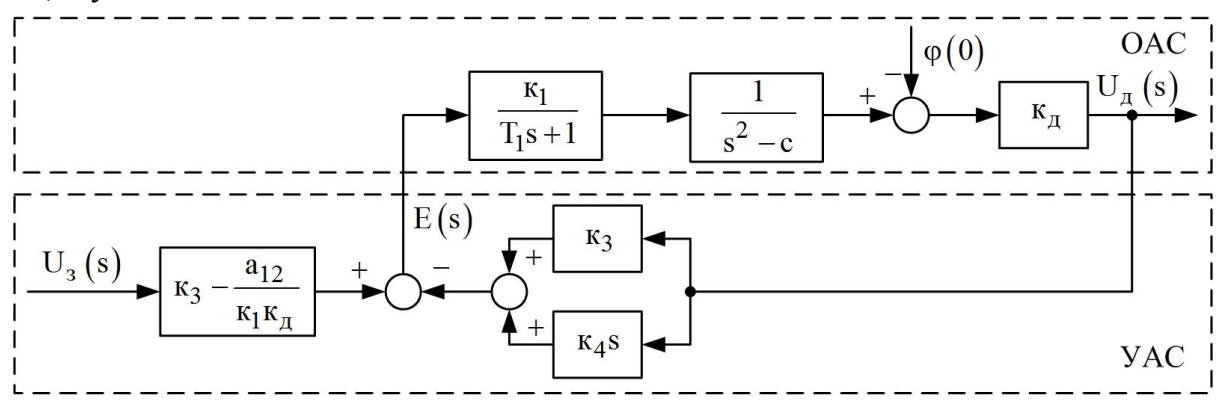

Рис. 6. Структурная схема модифицированной системы автоматической стабилизации 
Для проверки правильности процедуры синтеза необходимо оценить показатели качества переходного процесса. Известно несколько методов оценки качества переходных процессов. Самый простой заключается в использовании нормированных переходных характеристик, соответствующих оптимальным коэффициентам нормированной передаточной функции для интегрального критерия взвешенного модуля ошибки [3]. Зная численное значение $\omega_{n}=2,86$ можно определить время переходного процесса. Для используемого ступенчатого управляющего воздействия можно определить перерегулирование и точность в установившемся режиме.

Более продуктивный метод заключается в построении графика переходного процесса и определении его показателей качества. Построение графика переходного процесса связано с формированием изображения выходного сигнала системы $U_{\partial}(s)$ как реакции системы на ступенчатый входной сигнал. Полученное изображение представляет собой дробно-рациональную функцию. Для знаменателя этой функции вычисляются корни. Исходя из полученной структуры корней производится разложение изображения $U_{\partial}(s)$ на элементарные дроби с неизвестными значениями коэффициентов числителей.
В результате соответствующих преобразований вычисляются значения коэффициентов.

Для получения оригинала переходного процесса используется обратное преобразование Лапласа $u_{\partial}(t)=L^{-1}\left\{U_{\partial}(s)\right\}$. С помощью таблиц обратных преобразований Лапласа формируется аналитическое выражение переходного процесса для построения его графика. По графику переходного процесса вычисляются фактические показатели качества.

Анализ полученных показателей качества позволяет установить допущенные при синтезе ошибки, а в случае их отсутствия выяснить степень их соответствия желаемым показателям качества и наметить пути по дальнейшему практическому совершенствованию системы стабилизации неустойчивых состояний обратного маятника с винтовыми электроприводами.

Блок-схема технологического процесса синтеза. Представим в графическом виде технологический процесс синтеза посредством этапов и инструментальных средств (рис. 7). Процесс синтеза - итерационный процесс. Для упрощения графического представления технологии на блок-схеме не показаны обратные связи, вызванные контролем каждого этапа синтеза.

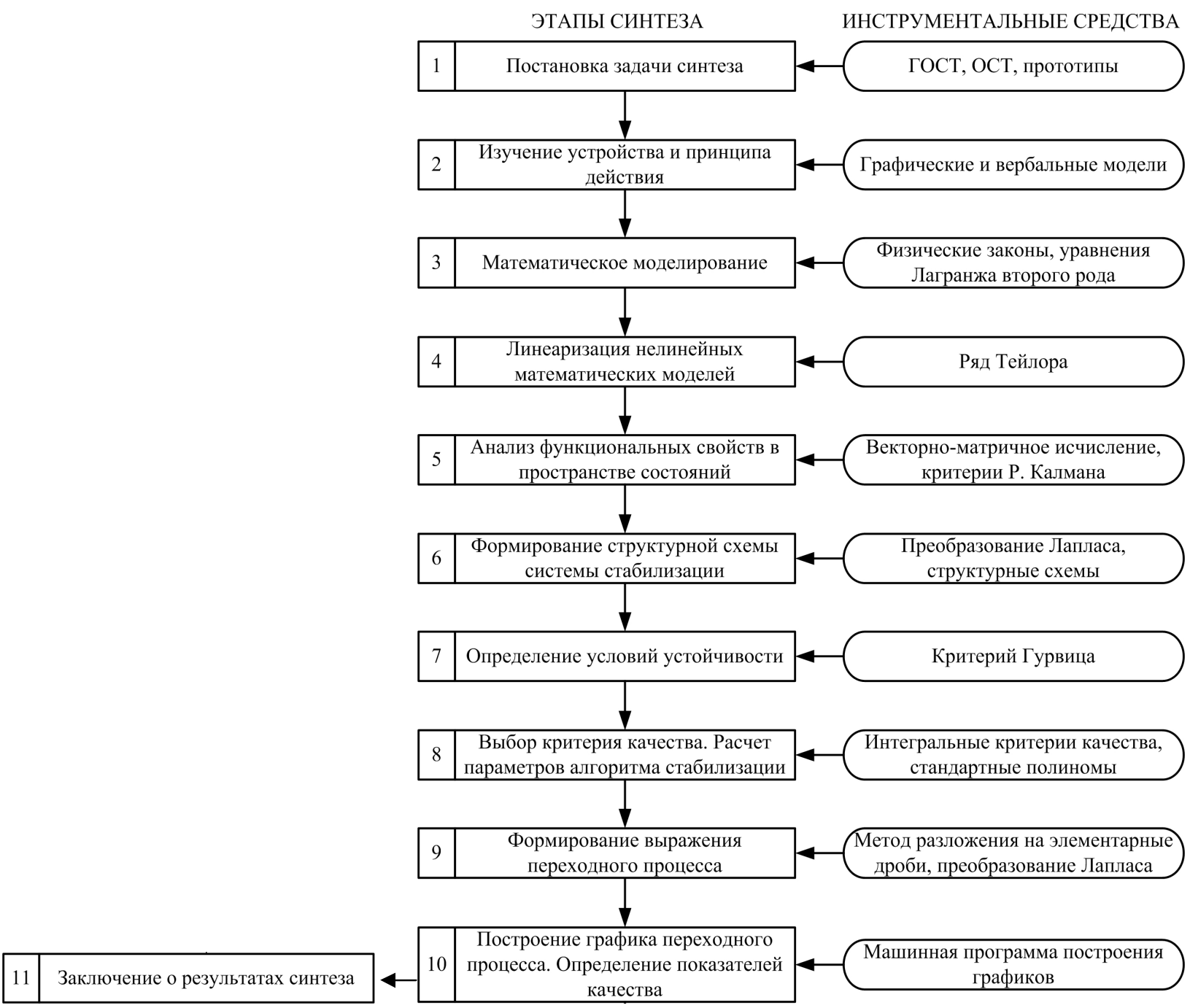

Рис. 7. Блок-схема технологического процесса синтеза 


\section{6. Пример параметрического синтеза алгоритма стабилизации обратного маятника}

В исследованиях был использован макетный образец обратного маятника с винтовыми электроприводами со следующими значениями коэффициентов передаточных функций ОАС: $\kappa_{1}=0,9 \frac{p a \partial}{B \cdot c^{2}}$; $T_{1}=0,2 c ; c=49 \frac{p a \partial}{c^{2}} ; \kappa_{\partial}=7,14 \frac{B}{p a \partial}$.

Из соотношений (42), получим:

$$
\omega_{n}=2,86 ; \kappa_{4}=2,07 ; \kappa_{3}=8,35 .
$$

Полученные значения удовлетворяют необходимым (35) и достаточным (36) условиям устойчивости.

Передаточная функция замкнутой системы при полученных значениях коэффициентов (43) будет иметь следующий вид:

$$
\Phi_{1}(s)=\frac{U_{\partial}(s)}{U_{3}^{\prime}(s)}=\frac{23,39}{s^{3}+5 s^{2}+17,59 s+23,39} .
$$

При ступенчатом задающем воздействии $U_{3}^{\prime}(s)=\frac{0,64}{s}$, соответствующем отклонению маятника на $5^{\circ}$, изображение выходного сигнала

$$
U_{\partial}(s)=\frac{15,03}{s\left(s^{3}+5 s^{2}+17,59 s+23,39\right)} .
$$

Характеристическому уравнению замкнутой системы удовлетворяют такие значения корней:

$$
s_{1}=-2,02 ; s_{2}=-1,49+j 3,06 ; s_{3}=-1,49-j 3,06 \text {. }
$$

Разложим изображение выходного сигнала на элементарные дроби:

$$
U_{\partial}(s)=\frac{A}{s}+\frac{B}{s+2,02}+\frac{D s+F}{s^{2}+2,98 s+11,52} .
$$

Выполнив соответствующие преобразования и вычисления, получим следующие значения коэффициентов:

$$
A=0,64 ; B=-0,37 ; D=-0,27 ; F=-1,55 .
$$

Применяя обратное преобразование к элементарным дробям, преобразованным к соответствующим табличным выражениям

$$
\begin{aligned}
& u_{\partial}(t)=L^{-1}\left\{\frac{0,64}{s}\right\}+L^{-1}\left\{\frac{-0,37}{s+2,02}\right\}+ \\
& +L^{-1}\left\{-0,27 \frac{s+1,49}{(s+1,49)^{2}+3,06^{2}}\right\}+
\end{aligned}
$$

$$
+L^{-1}\left\{-0,19 \cdot 3,06 /\left((s+1,49)^{2}+3,06^{2}\right)\right\}
$$

получим выражение переходного процесса:

$$
\begin{gathered}
u_{\partial}(t)=0,64-0,37 e^{-2,02 t}- \\
-0,27 e^{-1,49 t} \cos (3,06 t)-0,19 e^{-1,49 t} \sin (3,06 t) .
\end{gathered}
$$

С учетом коэффициента передачи предшествующего фильтра на вход системы нужно подать ступенчатое воздействие $u_{3}(t)=0,47 \cdot 1(t)$, чтобы отклонить маятник на $5^{\circ}$.

График переходного процесса представлен на рис. 8.

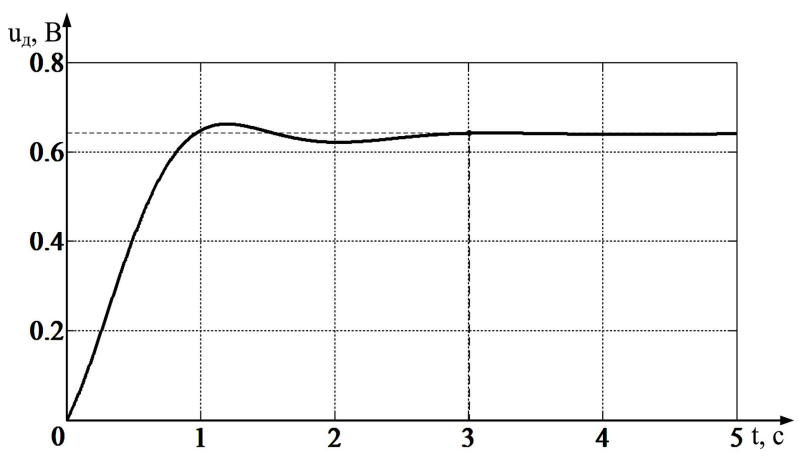

Рис. 8. График переходного процесса системы стабилизации

В установившемся режиме ошибка равна нулю. Время переходного процесса составляет - 3 с. Перерегулирование - 3,1\%. Переходный процесс соответствует минимальному значению интегрального критерия качества (38).

\section{Заключение}

В результате проведенных исследований возможности стабилизации неустойчивых состояний обратного маятника с винтовыми электроприводами удалось сформировать технологический процесс параметрического синтеза алгоритмов стабилизации. В процессе исследований были получены разнотипные модели обратного маятника как объекта автоматической стабилизации, отличающиеся от известных учетом динамических свойств винтовых электроприводов. Сформированы условия выбора параметров пропорционально-дифференциальной структуры алгоритмов стабилизации, обеспечивающие устойчивость и оптимальность переходного процесса по интегральному критерию взвешенного модуля ошибки.

Полученные результаты могут использоваться как в учебном процессе в разделах проектирования систем управления неустойчивых объектов, так и на этапах эскизного проектирования простейших автономных летательных аппаратов.

\section{СПИСОК ЛИТЕРАТУРЫ}

1. Боднер, В. А. Системы управления летательными аппаратами / В. А. Боднер. - М. : Машиностроение, 1973. -504 с.

2. Разоренов, Г. Н. Системы управления летательными аппаратами [Текст] : учеб. для вузов / Г. Н. Разоренов, Э. А. Бахрамов, Ю. Ф. Титов ; под ред. Г. Н. Разоренова. - М. : Машиностроение, 2003. - 584 с.

3. Формальский, А. М. Управление движением неустойчивых объектов [Текст] / А. М. Формальский. - М. : ФИЗМАТЛИТ, 2014. - 232 с. 
4. Кулик, А. С. Обеспечение отказоустойчивости систем управления статически неустойчивыми динамическими объектами [Текст] / А. С. Кулик, О. И. Гавриленко // Успехи совершенной радиоэлектроники. - 2004. - № 2. - С. $52-75$.

5. Федосов, Б. Т. Управление неустойчивыми объектами. Обратный маятник. [Электронный ресурс] / Б. Т. Федосов. Режим доступа: http: // model.exponenta.ru/bt/bt_152_Inv Pend_control_1.htm. - 28.07.2009 г.

6. Ряжских, В. И. Стабилизация обратного маятника на двухколесном транспортном средстве [Текст] / В. И. Ряжских, М. Е. Семенов, А. Г. Рукавицын, О. И. Канищева, А. А. Демчук, П. А. Мелешенко // Вестн. Южно-Ур. гос. ун-та. Серия «Математика. Механика. Физика». - 2017. - Т. 9, № 3. - С. 41 - 50.

7. Кулик, А. С. Методы моделирования объектов автоматического управления [Текст] : учеб. пособие / А. С. Кулик, С. Н. Пасичник. - Харьков : Нац. аэрокосм. ун-т им. Н. Е. Жуковского «ХАИ», 2018. - 168 с.

8. Коваленко А.А. Сучасний стан та тенденції розвитку комп'ютерних систем об'єктів критичного застосування / А.А. Коваленко, Г.А. Кучук // Системи управління, навігації та зв'язку. - Полтава . ПНТУ, 2018. - Вип. 1(47). - С. 110-113.

9. Дорф, Р. Современные системы управления [Текст] : пер. с англ. / Р. Дорф, Р. Бишоп. - М. : Лаборатория базовых знаний, 2002. -832 с.

Рецензент: д-р техн. наук, проф. О. В. Козелков, Державний університет телекомунікацій, Київ Received (Надійшла) 29.10.2018

Accepted for publication (Прийнята до друку) 09.01.2019

\section{Стабілізація нестійких станів зворотного маятника з гвинтовими електроприводами}

\section{А. С. Кулік, К. Ю. Дергачов, С. М. Пасічник, Ю. О. Немшилов}

Предметом вивчення в статті $є$ технологічний процес аналітичного синтезу алгоритмів стабілізації нестійких станів зворотного маятника з гвинтовими електроприводами. Метою $\epsilon$ розробка технології оптимального аналітичного синтезу алгоритмів стабілізації нестійких станів зворотного маятника з гвинтовими електроприводами. Завдання: зформувати фізичну модель зворотного маятника з гвинтовими електроприводами. Розробити за допомогою Лагранжевого формалізму нелінійну математичну модель зворотного маятника з гвинтовими електроприводами як об'єкту автоматичної стабілізації. Побудувати лінеаризовану математичну модель об'єкту автоматичної стабілізації у просторі станів. Проаналізувати функціональні властивості об'єкту автоматичної стабілізації. Сформувати структурні схеми системи автоматичної стабілізації станів зворотного маятника 3 гвинтовими електроприводами. Отримати аналітичні співвідношення для обчислення оптимальних значень параметрів алгоритмів стабілізації нестійких станів зворотного маятника 3 гвинтовими електроприводами. Синтезувати для конкретного зворотного маятника з гвинтовими електроприводами алгоритм оптимальної стабілізації кутового положення. Використовуваними методами є: метод Лагранжа, аналітичної лінеаризації, простору станів, перетворення Лапласа. Отримані такі результати. Сформовано технологічний процес оптимального аналітичного синтезу алгоритмів стабілізації нестійких станів зворотного маятника з гвинтовими електроприводами. На конкретному числовому прикладі проілюстровано продуктивність запропонованого процесу аналітичного конструювання та інструментальних засобів для параметричного синтезу алгоритмів стабілізації зворотного маятника з гвинтовими електроприводами. Висновки. Наукова новизна отриманих результатів полягає в такому: отримано різнотипні моделі зворотного маятника з гвинтовими електроприводами як об'єкту автоматичної стабілізації, що відрізняються від відомих урахуванням динамічних властивостей гвинтових електроприводів, які створюють керуючі моменти стабілізації. Сформовано умови вибору параметрів пропорційно-диференціальної структури алгоритмів стабілізації для забезпечення стійкості та оптимальності перехідного процесу замкненої системи стабілізації, що відрізняються від відомих специфікою формування керуючих моментів стабілізації за інтегральним критерієм зваженого модулю похибки.

Ключов і слов а : зворотний маятник, гвинтовий електропривод, об’єкт автоматичної стабілізації, алгоритм стабілізації, аналітичний синтез, стабілізація нестійких станів, інтегральний критерій зваженого модулю похибки.

\section{Unstable states stabilization of the reverse pendulum with screw actuators}

A. Kulik, K. Dergachov, S. Pasichnik, Yu. Nemshilov

The subject of study in the article is the technological process for the analytical synthesis of stabilization algorithms for unstable states of a reverse pendulum with screw actuators. The purpose is to develop the technology of optimal analytical synthesis of stabilization algorithms for unstable states of a reverse pendulum with screw actuators. Tasks: to form the physical model of a reverse pendulum with screw actuators. To develop using the Lagrangian formalism nonlinear mathematical model of the reverse pendulum with screw electric actuators as the automatic stabilization object. Build the linearized mathematical model of the automatic stabilization object in the state space. To analyze the functional properties of the automatic stabilization object. To form the structural schemes of the states' stabilization automatic system for the reverse pendulum with screw actuators. To obtain analytical relations for calculating the optimal parameters values of the stabilization algorithms for unstable states of the reverse pendulum with screw actuators. To synthesize the optimal stabilization algorithm of the angular position for the specific reverse pendulum with screw electric actuators. The methods used are: Lagrange's method, analytic linearization, state space, Laplace's transformation. The following results were obtained. A technological process of optimal analytical synthesis of stabilization algorithms for unstable states of the reverse pendulum with screw actuators has been formed. The specific numerical example illustrates the productivity of the proposed process of analytical design and tools for the parametric synthesis of the stabilization algorithms for the reverse pendulum with screw actuators. Conclusions. The scientific novelty of the results is as follows: various models of the reverse pendulum with screw actuators are obtained as the automatic stabilization object, differing from those known for taking into account the dynamic properties of screw actuators that create control stabilization moments. The conditions for choosing the parameters of the proportional-differential structure of stabilization algorithms are formed to ensure the stability and optimality of the transition process of the close-loop stabilization system that differ from those known by the formation specifics of the stabilization control moments by the integral criterion of a weighted error modulus.

Keywords: reverse pendulum, screw actuator, automatic stabilization object, stabilization algorithm, analytical synthesis, unstable states stabilization, integral criterion for a weighted error modulus. 